\title{
Supporting Information: Photoinduced Carrier Dynamics at the Interface of Pentacene and Molybdenum Disulfide
}

\author{
Xiao-Ying Xie, Xiang-Yang Liu, Qiu Fang ${ }^{*}$, Wei-Hai Fang, and Ganglong Cui ${ }^{*}$
}

Key Laboratory of Theoretical and Computational Photochemistry, Ministry of Education, Chemistry College, Beijing Normal University, Beijing 100875, China

E-mail: fangqiu917@bnu.edu.cn; ganglong.cui@bnu.edu.cn

\section{Table of Contents}

\section{Tables}

Table S1: Relative energies of pentacene/MoS 2 models calculated at PBE+D3 and HSE06 levels.

Table S2: Characters (in percentage) of four adiabatic states i.e. states $515-517$, and state-520 located on the $\mathrm{MoS}_{2}$ monolayer.

\section{Figures}

Figure S1. PBE+D3 optimized pentacene@MoS2 structures with a "standing" pentacene molecule.

Figure S2. DOS of pentacene@MoS 2 (parallel model).

Figure S3. DOS of pentacene@MoS (vertical_1 model).

Figure S4. DOS of pentacene@MoS (vertical_2 model).

Figure S5. Calculated PDOS of pentacene@MoS2 in parallel model at HSE06 level conducted by VASP.

Figure S6. Calculated spatial distributions of six adiabatic states from states 515 to 520 involved in the interfacial hole transfer and the electron-hole recombination dynamics of pentacene@MoS 2 at PBE+D3 level.

Figure S7. DOS of isolated pentacene and $\mathrm{MOS}_{2}$ calculated at the HSE06 level.

Figure S8. Vibrational spectrum from the Fourier transformation of the time-dependent energy of state- 516 .

Figure S9. Time-dependent state populations of states 516 to 519 and the corresponding time-dependent hole amount localized on the MoS2 fragment in the nonadiabatic dynamics simulations for the interfacial hole transfer starting from state-516.

Figure S10. Adiabatic and nonadiabatic contributions for the interfacial hole transfer between the $\mathrm{MoS}_{2}$ monolayer and the pentacene molecule.

Figure S11. Spatial superposition of ground- and excited-state geometries. 


\section{Tables}

Table S1: Relative Energies of Pentacene/MoS 2 Models Calculated at PBE+D3 and HSE06 Levels.

\begin{tabular}{|c|c|c|}
\hline \multirow{2}{*}{ Model } & \multicolumn{2}{|c|}{ Relative energy (eV) } \\
\cline { 2 - 3 } & PBE+D3 & HSE06 \\
\hline parallel & 0.00 & 0.00 \\
\hline vertical_1 & 0.87 & 1.03 \\
\hline vertical_2 & 1.45 & 1.60 \\
\hline
\end{tabular}

Table S2: Characters (in Percentage) of Four Adiabatic States i.e. States 515-517, and State-520 Located on the $\mathrm{MoS}_{2}$ Monolayer.

\begin{tabular}{|c|c|c|c|c|}
\hline Mo & State-515 & State-516 & State-517 & State-520 \\
\hline $\mathbf{d}_{\mathbf{x y}}$ & 36.8 & 37.0 & 0 & 0 \\
\hline $\mathbf{d}_{\mathbf{y z}}$ & 0 & 0 & 0 & 0 \\
\hline $\mathbf{d}_{\mathbf{z 2}}$ & 0 & 0 & 69.6 & 81.7 \\
\hline $\mathbf{d}_{\mathbf{x z}}$ & 0 & 0 & 0 & 0 \\
\hline $\mathbf{d}_{\mathbf{x 2}-\mathbf{y} \mathbf{2}}$ & 36.8 & 37.1 & 0 & 0 \\
\hline
\end{tabular}

\section{Figures}

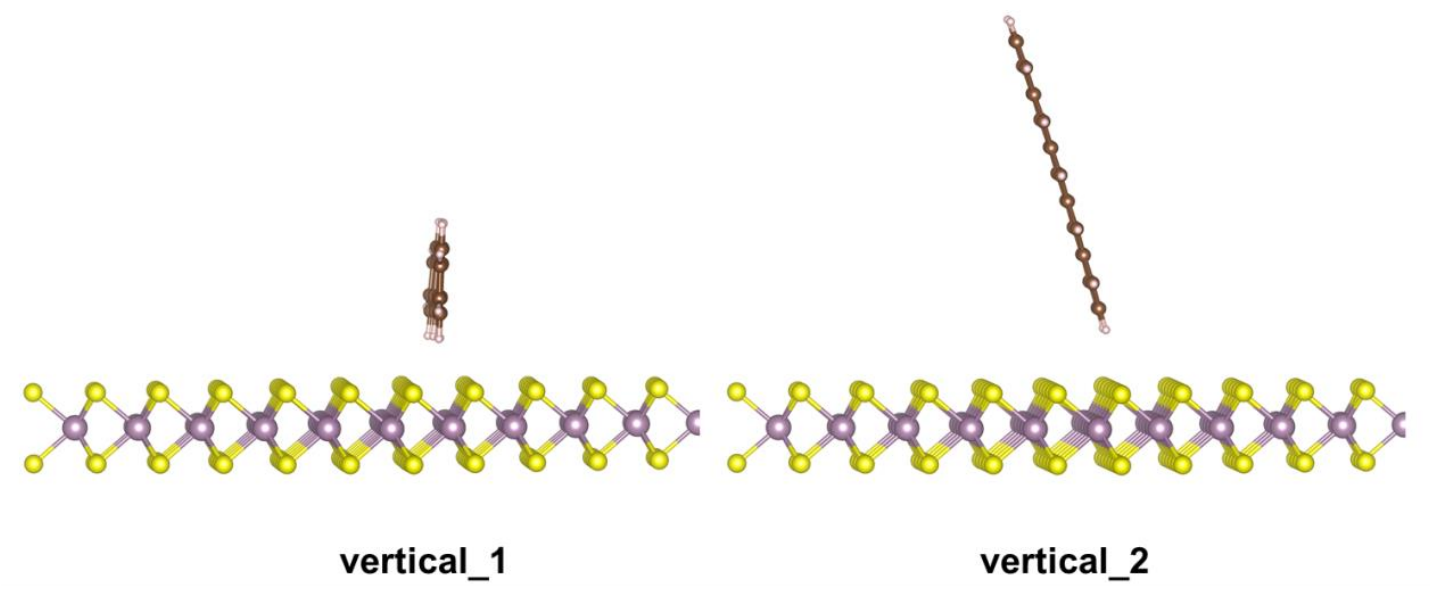

Figure S1. PBE+D3 optimized pentacene@MoS 2 structures with a "standing" pentacene molecule (models: vertical_1 and vertical_2). 

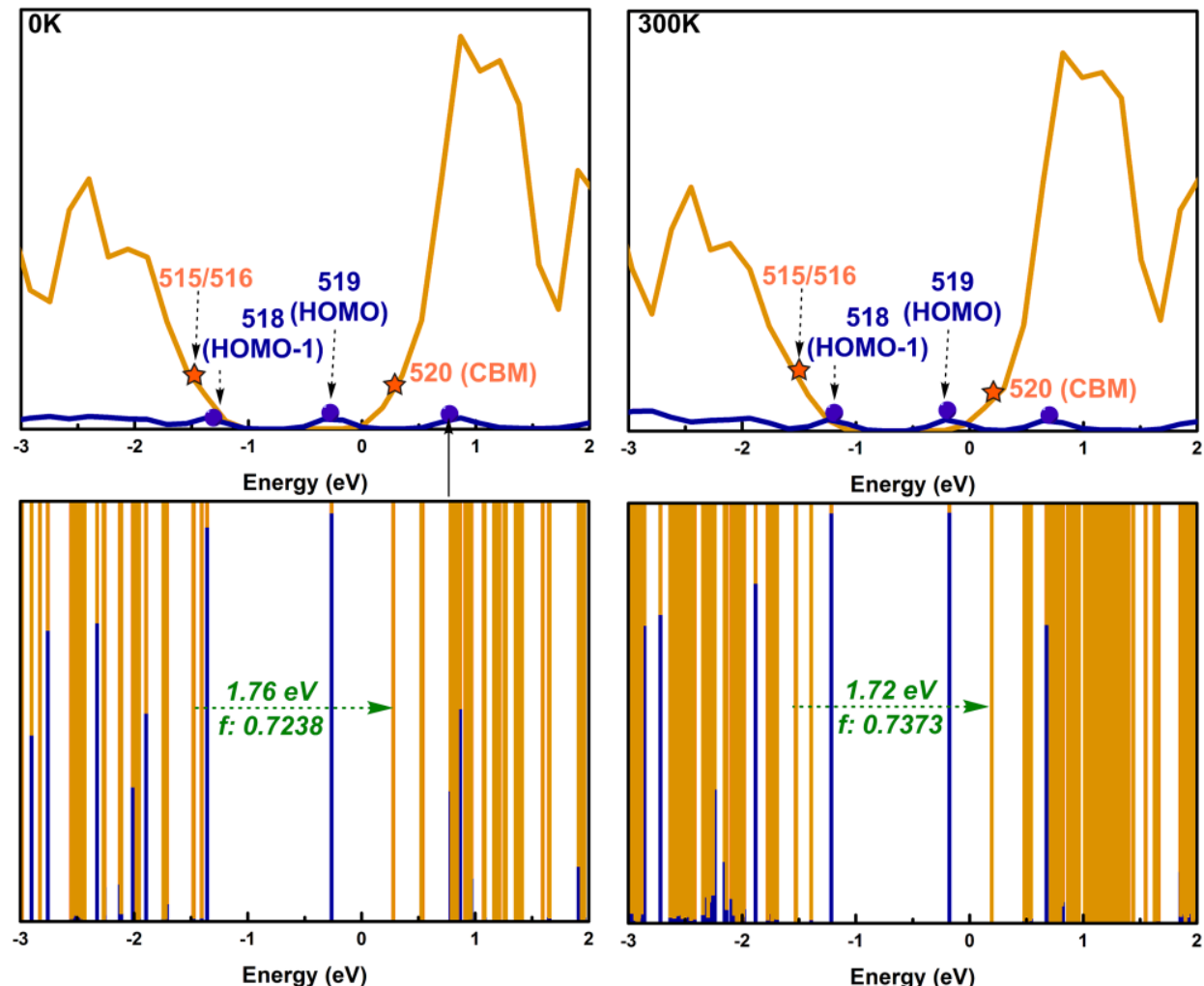

Figure S2. (top) DOS and PDOS of pentacene@MoS 2 (parallel model) at (left) $0 \mathrm{~K}$ and (right) $300 \mathrm{~K}$ calculated at PBE+D3 level; (bottom) the contributions of $\mathrm{MoS}_{2}$ (orange) and pentacene (blue) fragments to each adiabatic state.
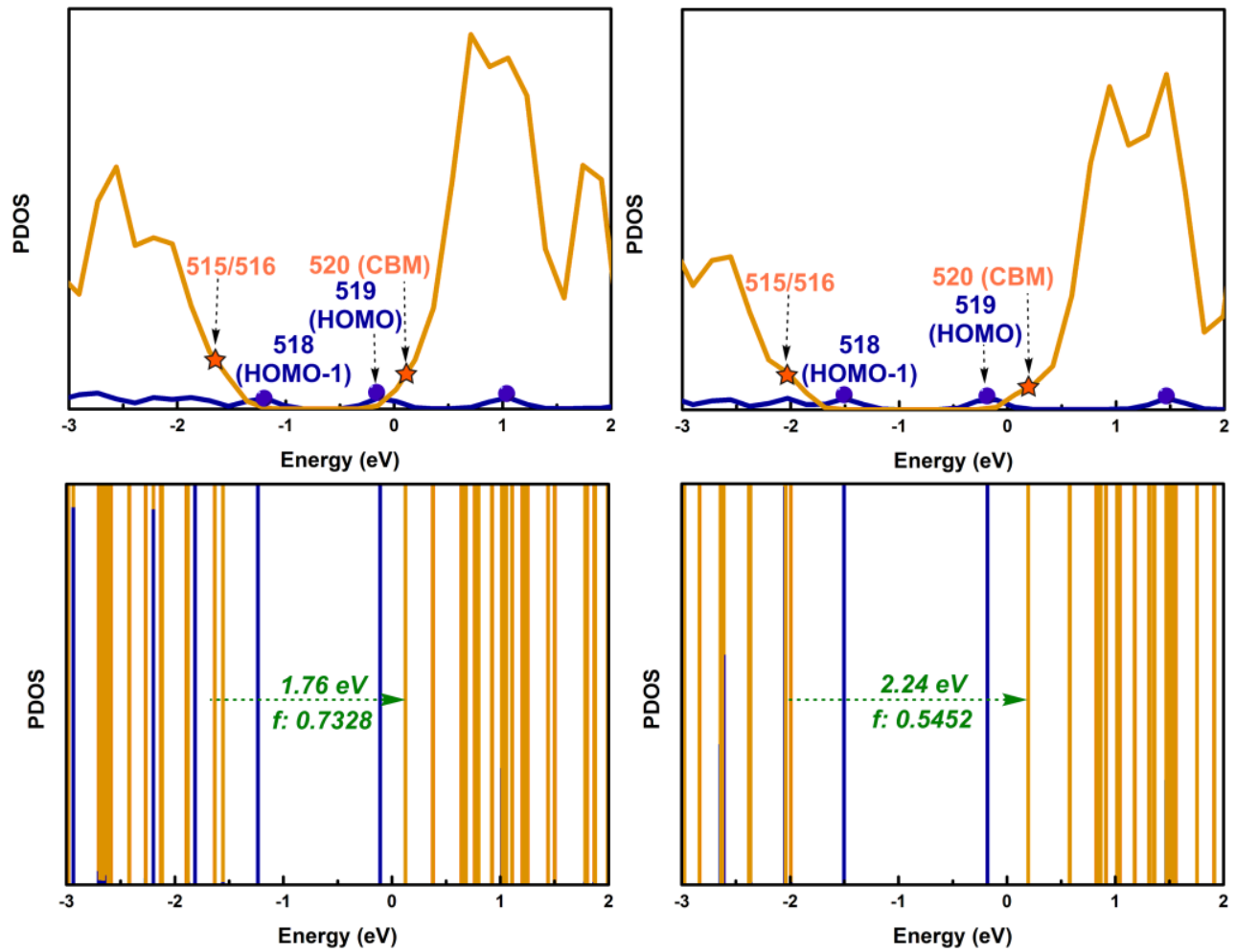

Figure S3. (top) DOS and PDOS of pentacene@MoS 2 (vertical_1 model) at (left) PBE+D3 level and (right) HSE06 level; (bottom) contributions of $\mathrm{MoS}_{2}$ (orange) and pentacene (blue) fragments to each adiabatic state. 

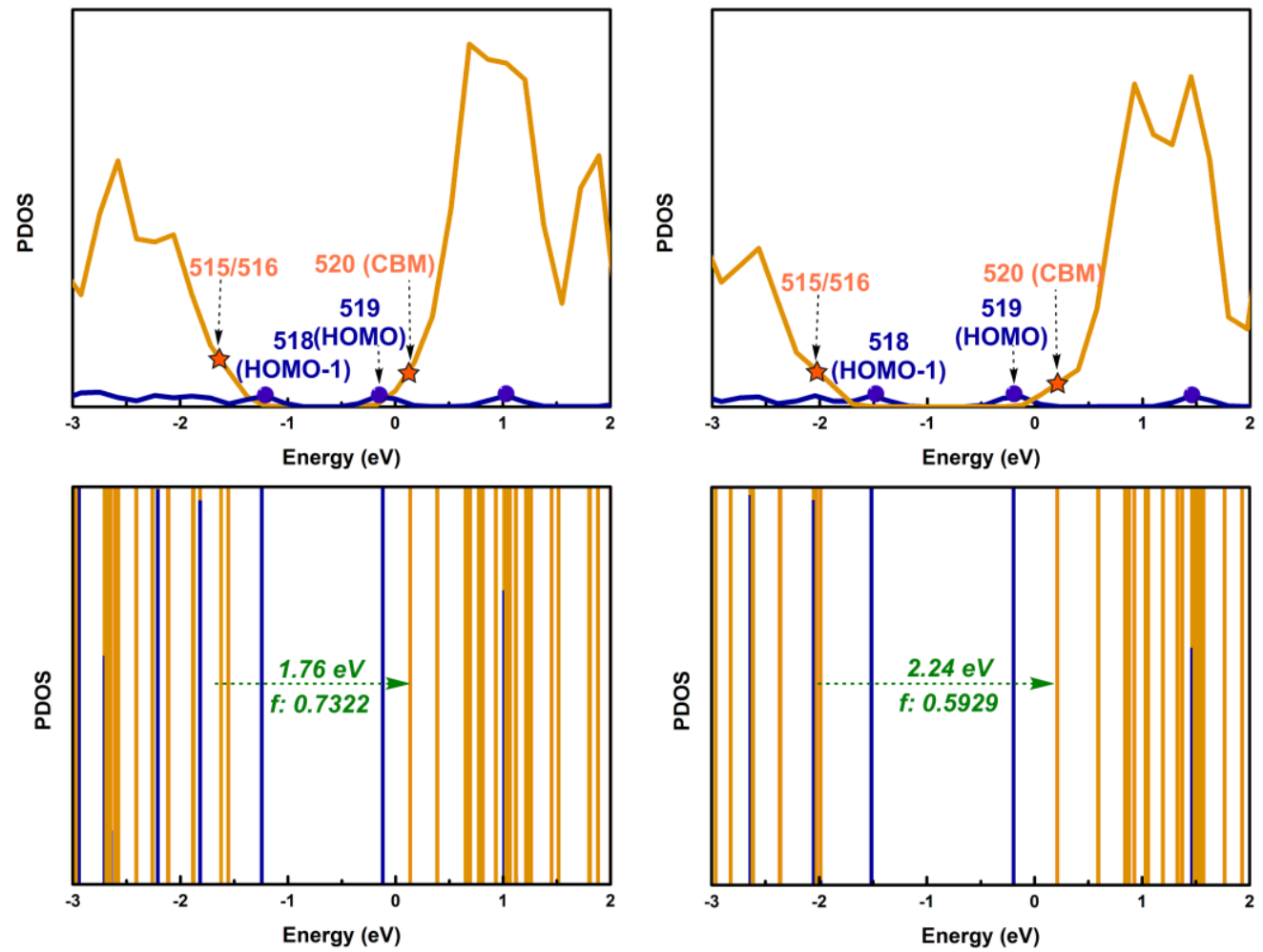

Figure S4. (top) DOS and PDOS of pentacene@MoS 2 (vertical_2 model) at (left) PBE+D3 level and (right) HSEO6 level; (bottom) contributions of $\mathrm{MoS}_{2}$ (orange) and pentacene (blue) fragments to each adiabatic state.

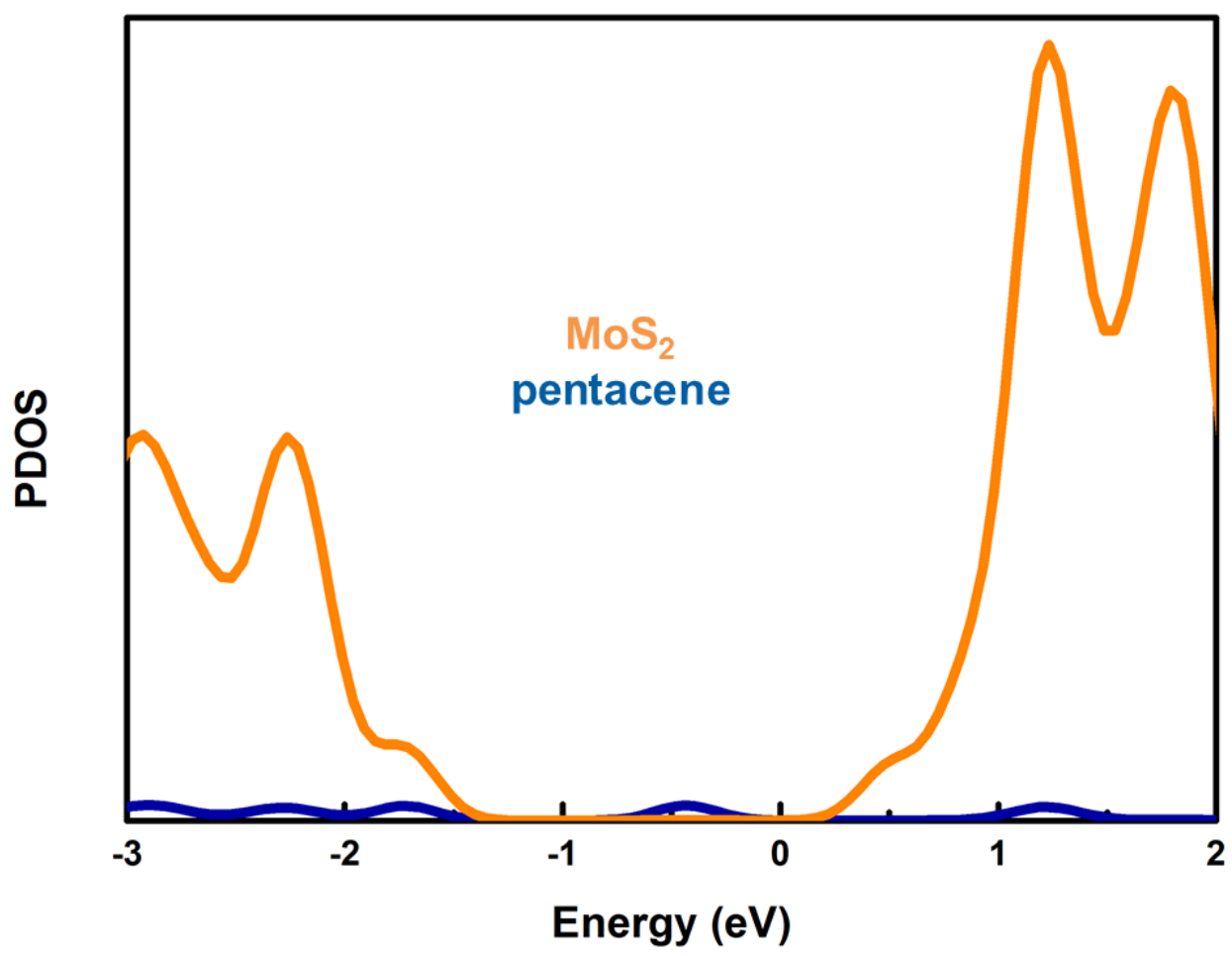

Figure S5. Calculated PDOS of pentacene@MoS 2 in parallel model at HSE06 level conducted by VASP. 


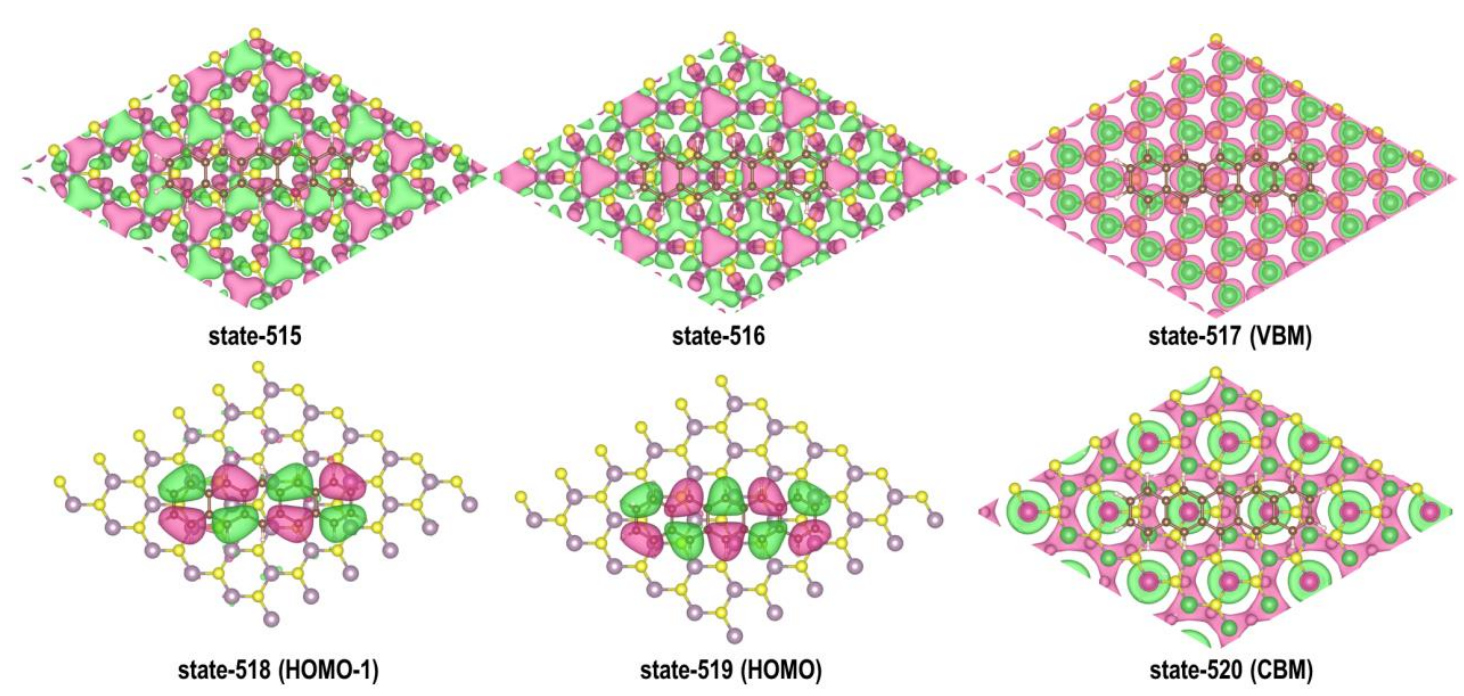

Figure S6. Calculated spatial distributions of six adiabatic states from states 515 to 520 involved in the interfacial hole transfer and the electron-hole recombination dynamics of pentacene@MoS 2 at PBE+D3 level.
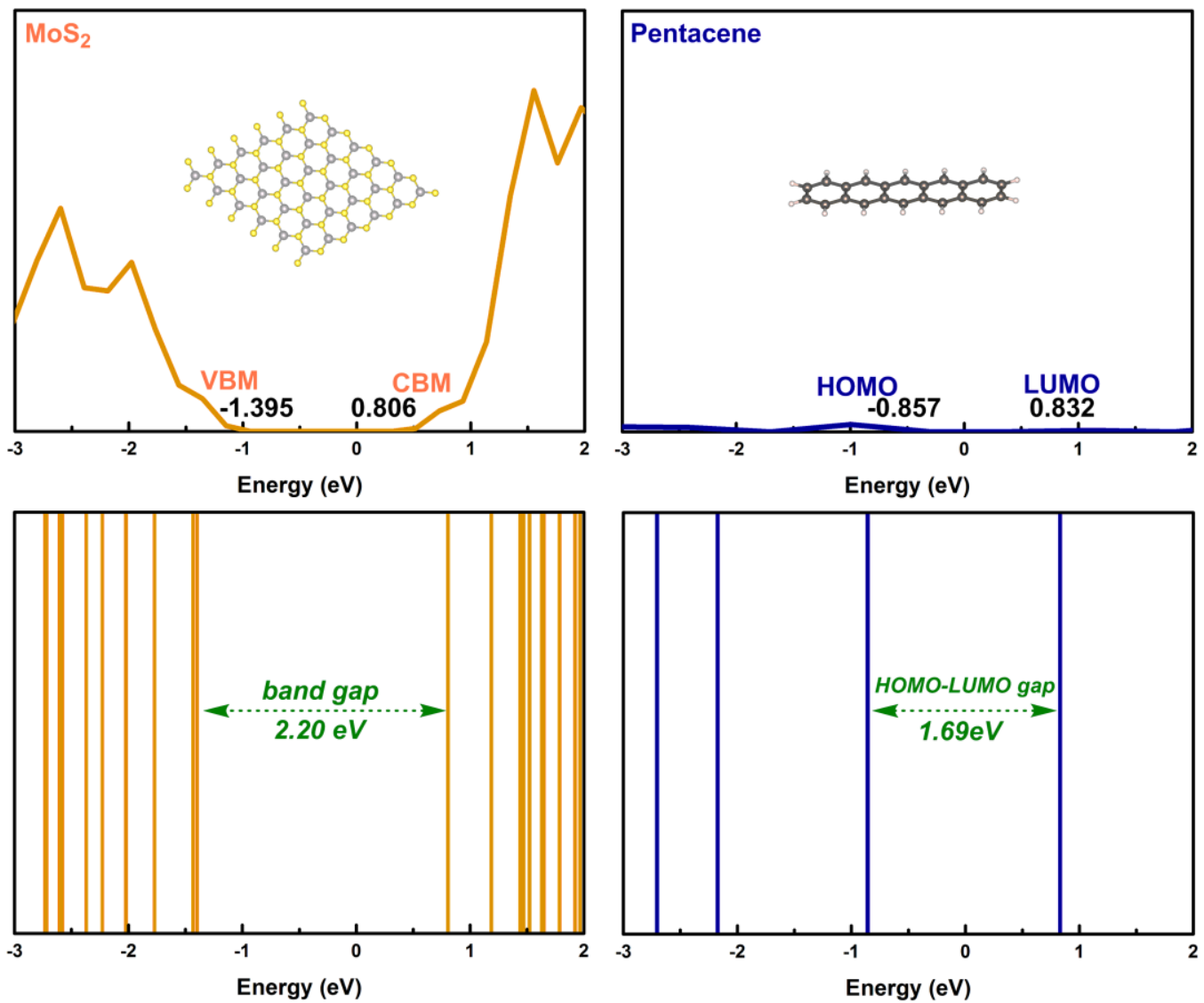

Figure S7. DOS of isolated pentacene (left) and $\mathrm{MoS}_{2}$ (right) calculated at the HSE06 level. 


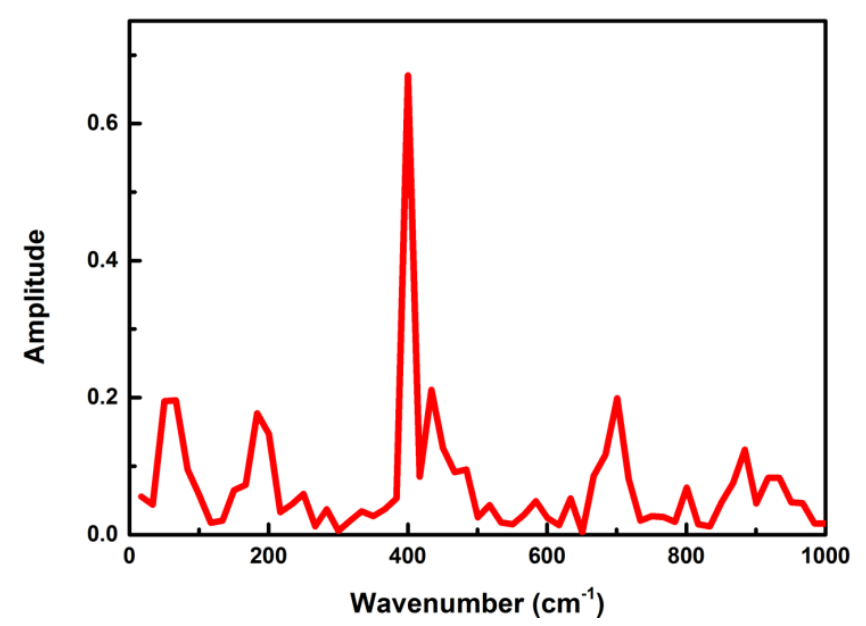

Figure S8. Vibrational spectrum from the Fourier transformation of the time-dependent energy of state- 516 .
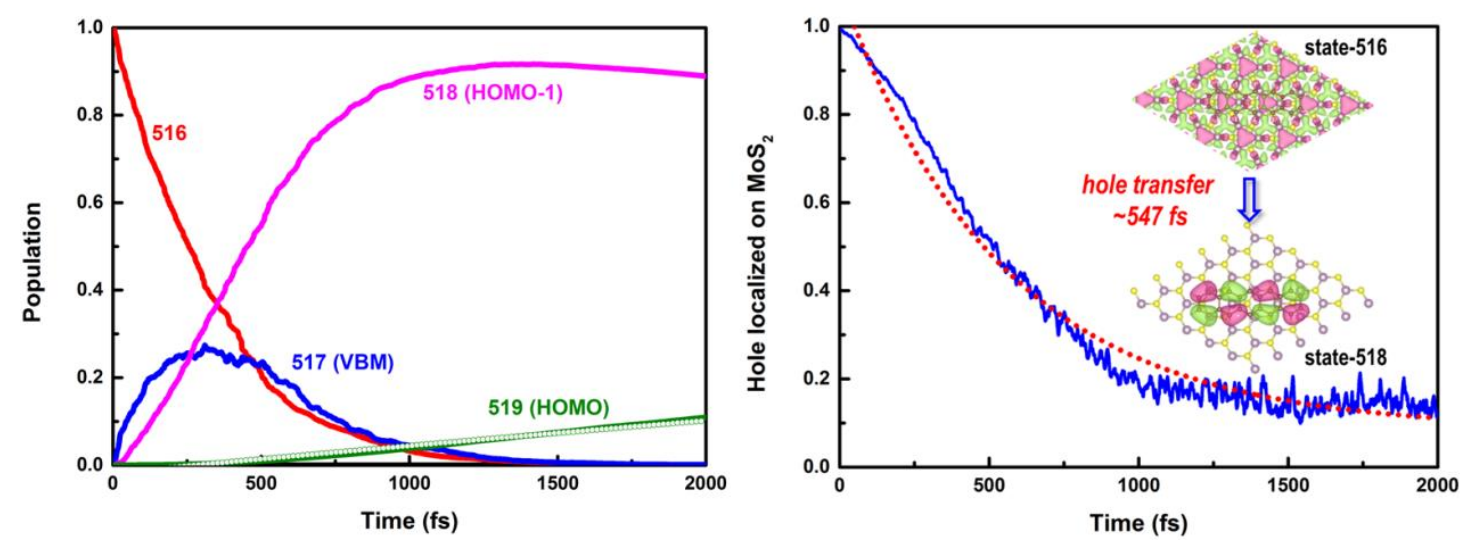

Figure S9. (left) Time-dependent state populations of states 516 to 519 and (right) the corresponding time-dependent hole amount localized on the $\mathrm{MoS}_{2}$ fragment in the nonadiabatic dynamics simulations for the interfacial hole transfer starting from state- 516 .
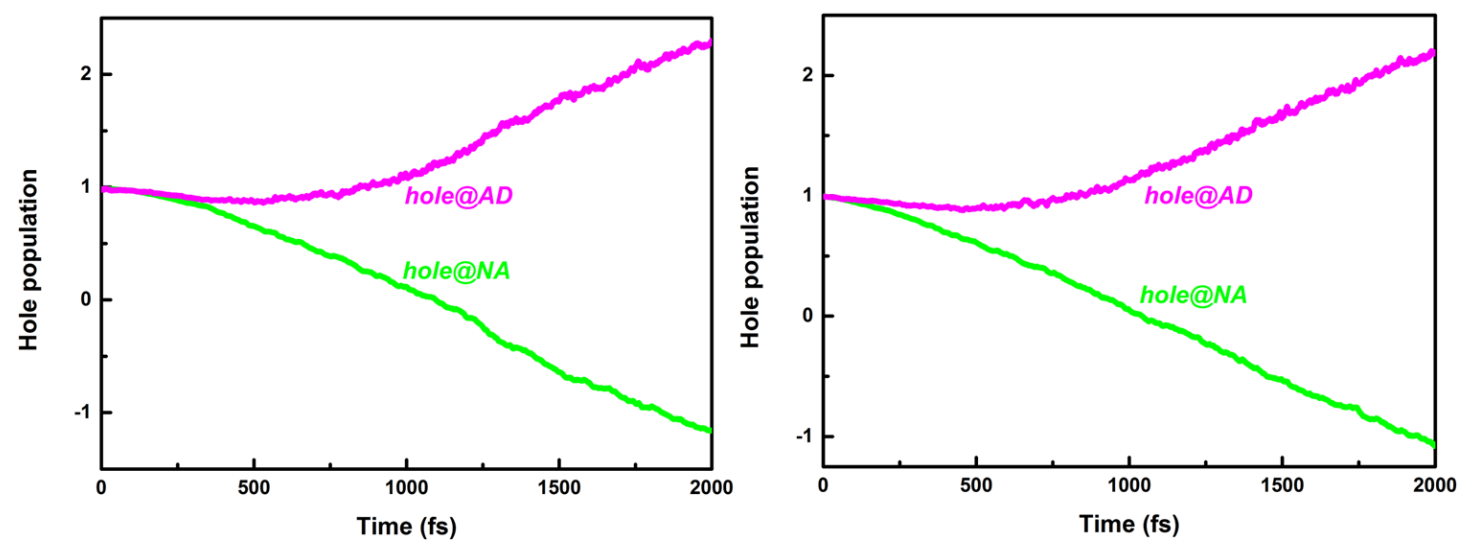

Figure S10. Adiabatic and nonadiabatic contributions to the interfacial hole transfer from $\mathrm{MoS}_{2}$ to pentacene from (left) state-515 and (right) state-516. It is clear that nonadiabatic one plays a favorable role while adiabatic one is a little against the hole transfer. Nonetheless, the hole transfer is totally favorable. 


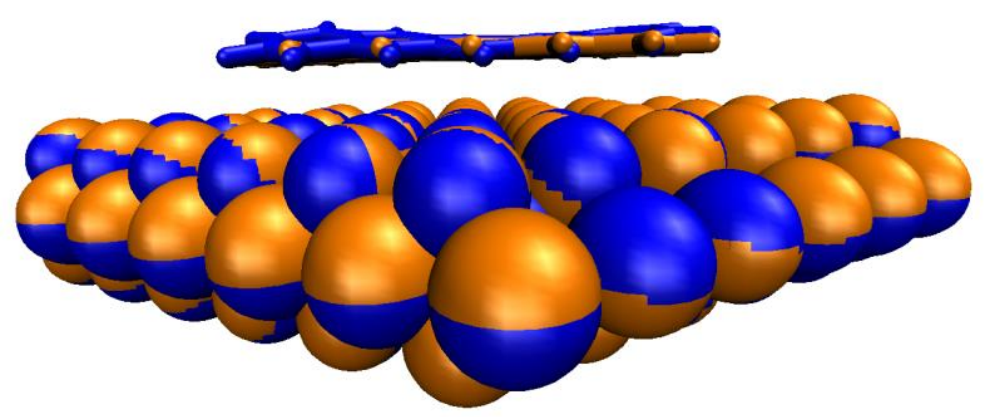

Figure S11. Spatial superposition of optimized ground state (orange) and charge-transfer excited state (blue) geometries. The latter is optimized using the constrained density functional theory (CDFT) method implemented in CP2K by enforcing an electron localized on pentacene while another one on $\mathrm{MoS}_{2}$. 